

\section{CLOZAPINE-INDUCED DOUBLE INCONTINENCE}

Sir,

Double incontinence (bowel and bladder incontinence) is an uncommon and underreported adverse effect of clozapine, which has been frequently associated with urinary incontinence. A case of clozapine-induced double incontinence is described, and the possible pathophysiological mechanism is discussed

A 23-year-old Indian male, diagnosed with schizophrenia of 16 months' duration, had been resistant to a trial of conventional antipsychotics. Subsequently, he was started on clozapine 25 $\mathrm{mg} /$ day, which was increased to $75 \mathrm{mg} /$ day over a period of 2 weeks. Three weeks after starting clozapine, he developed bowel and bladder incontinence. While he had bowel incontinence almost daily during the afternoon when he was awake, the bladder incontinence was only nocturnal. It is noteworthy that bowel and bladder incontinence never occurred simultaneously. He discontinued clozapine on his own, resulting in amelioration of the symptoms. However, the double incontinence returned on restarting clozapine. He was seen by an internist who ruled out any medical etiology for the incontinence. He had no prior history of incontinence. A head CT and an EEG were read as normal. Clozapine was again discontinued, and the double incontinence resolved completely within the next two days. Subsequently, he was treated with risperidone $2 \mathrm{mg} /$ day without any recurrence of the incontinence.

On the Naranjo probability scale, the adverse drug reaction probability score for this patient to develop clozapine-induced double incontinence was nine, suggesting a definitive adverse reaction due to clozapine. ${ }^{[1]}$ A PubMed search yielded no reports of clozapine-induced double incontinence. However, there is a single report of this adverse effect with olanzapine. ${ }^{[2]}$ Reports of abnormalities of the adrenergic system in patients with idiopathic fecal incontinence provide insights into the pathophysiology of clozapine-induced incontinence. ${ }^{[3]}$ A recent study showed that topical phenylephrine, a selective $\alpha_{1}$-adrenergic agonist, increased the resting tone of the internal anal sphincter in patients of fecal incontinence. ${ }^{[4]}$ Clozapine has a potent $\alpha_{1}$-adrenergic antagonist effect, which has been hypothesized to cause bladder incontinence; and the same can be extrapolated to bowel incontinence. ${ }^{[5}$ Alpha-adrenergic agents have been used to successfully treat clozapine-induced urinary incontinence, ${ }^{[5]}$ and it would be interesting to study the efficacy of this treatment in patients with clozapine-induced bowel incontinence.

D. N. MENDHEKAR, H. S. DUGGAL ${ }^{1}$ Neuropsychiatry Clinic, Pratap N agar M etro, Delhi-7, India; ${ }^{1}$ Psychiatry U nit, H errick M emorial Hospital, Tecumseh, Mich., USA Correspondence: Dr. D. N. Mendhekar, 10867 Pratap Nagar, Near Metro Pilla 129. Delhi - 110007 , India. E-mail: dnmendhekar@vsn!net

\section{REFERENCES}

1. Naranjo CA, Busto $U$, Sellers EM, Sandor $P$, Ruiz I, Roberts EA, et al. A method for estimating the probability of adverse drug reaction. Clin Pharmacol Ther 1981;30:239-45.

2. Sagar R, Varghese ST, Balhara YP. Olanzapine- induced double incontinence. Indian J Med Sci 2005;59:163-4.

3. Speakman CT, Hoyle CH, Kamin MA, Henry MM, Nicholls RJ, Burnstock G. Adrenergic control of the internal anal sphincter is abnormal in patients with idiopathic fecal incontinence. Br J Surg 1990;77:1342-4.

4. Cheetham MJ, Kamm MA, Phillips RK. Topical phenylephrine increases anal canal resting pressure in patients with faecal incontinence. Gut 2001;48:356-9.

5. Fuller MA, Borovicka MC, Jaskiw GE, Simo MR, Kwon K, Konicki PE. Clozapine-induced urinary incontinence: Incidence and treatment with ephedrine. J Clin Psychiatry 1996;57:5148. 